Fizika Nizkikh Temperatur, 1998, v. 24, No 8, p. 807-808

Letters to the Editor

\title{
Acoustically stimulated phase transition and low temperature optical spectra in $\mathrm{Pbl}_{2}$ crystal
}

\author{
M. M. Bilyi, I. S. Gorban, I. M. Dmitruk, I. M. Salivonov, and I. V. Ostrovskii \\ Physical Faculty, Kiev Taras Shevchenko University, 6 Akademic Glushkov Prs., 252127, Kiev, Ukraine \\ Received April 9, 1998 \\ The influence of intensive ultrasonic wave on the crystal structure of $\mathrm{PbI}_{2}$ was investigated. \\ Acoustically stimulated phase transition have been observed for the first time. \\ PACS: $64.70 . K b, 71.35 .-y$
}

$\mathrm{PbI} 2$ is a layered crystal and it consists of strongly (ionic forces) bounded layers. Layers interact with each other by the weak Van-der-Vaals forces. Many polytypes of PbI2 exist because of different possible orders of a layers package. As a result of weakness of interlayer forces, the phase polytype transitions are possible under the pressure or temperature change. Such a transition $2 \mathrm{H}$ $\operatorname{PbI} 2\left(D_{3 d}^{3}\right) \rightarrow 4 H-\operatorname{PbI} 2\left(C_{6 v}^{4}\right) \quad[1]$ can be observed under heating up to $130{ }^{\circ} \mathrm{C}$. The reverse transition takes place because of a metastable character of the $4 \mathrm{H}$ polytype phase after 1-2 months. Under the hydrostatic pressure an another type of phase transitions - polymorphous transitions - were observed. In such a type of the transition not only the layers orientation but the structure of layers are being changed [2].

The aim of this work is to study an influence of an ultrasonic wave on the $\mathrm{PbI}_{2}$ structure. We studied the transformations of the simplest and the most stable $2 H$ polytype single crystals after the exposure to the acoustical wave of the $2.25 \mathrm{MHz}$ frequency and the intensity about $1 \mathrm{~W} / \mathrm{cm}^{2}$. The PZT-type piezoceramic transducer was used to excite an ultrasonic wave in the sample. The maximum amplitude of rf-voltage applied to transducer $V_{p p}$ was $40 \mathrm{~V}$. The direction of the ultrasonic wave propagation was perpendicular to the crystal layers. A nondestructive study of crystal structure was performed by means of optical methods: reflectance and photoluminescence (PL), the low temperature spectroscopy and the Raman spectroscopy. We also measured acoustical noises (an acoustic emission, $\mathrm{AE}$ ) from our sample, the frequency range of measurements was 0.02 to $0.5 \mathrm{MHz}$.
The optical reflection spectra at the low temperature $(T=1.4 \mathrm{~K})$ were measured before and after the acoustical irradiation are presented on Fig. 1. One can see the $2 \mathrm{~nm}$ shift of a polariton reflection curve after the acoustical irradiation. This fact reflects the alteration of band width caused by the phase transformation: $2 \mathrm{H}-\mathrm{PbI}_{2} \rightarrow 4 \mathrm{H}-\mathrm{PbI}_{2}$. The same shift was observed in photoluminescence spectra (Fig. 2). The initial spectrum of $\mathrm{PbI}_{2}-2 \mathrm{H}$ (curve 1 ) has the emission bands of free excitons (FE) $496.5 \mathrm{~nm}$, excitons localized on impurities or defects (LE) $497.4 \mathrm{~nm}$, and the lower energy so called $K$-series. After the ultrasonic action a PL spectrum corresponds to the $4 H-\mathrm{PbI}_{2} \mathrm{PL}$ spectrum except the weak band at $497.4 \mathrm{~nm}$ which is due to the residue of the $2 H$ polytype. The $K$-series disappearing proves the existence of a phase transition, because this series is absent in the PL spectrum of the $4 H$ polytype. This conclusion is confirmed with Raman spectra measurements.

Type of the AE signal, its kinetics reflects the changes in a defect structure inside the sample initiated by the ultrasonic wave. The AE appeared when the ultrasound reached a certain threshold amplitude (rf-voltage $V_{p p}=24 \mathrm{~V}$ ). Under the ultrasound irradiation the intensity of $\mathrm{AE}$ signals increased with $V_{p p}$ increasing up to $40 \mathrm{~V}$. Then, under $V_{p p}=40 \mathrm{~V}=$ const, an evolution of the $\mathrm{AE}$ intensity in time achieved the maximum value and then decreased to zero. It lasted approximately 10 min. After the switching off the acoustic irradiation and following next increasing of $V_{p p}$, the AE reappeared at $V_{p p}>24 \mathrm{~V}$, lasted $10 \mathrm{~min}$ and then disappeared under the constant $V_{p p}$. Such a behavi- 


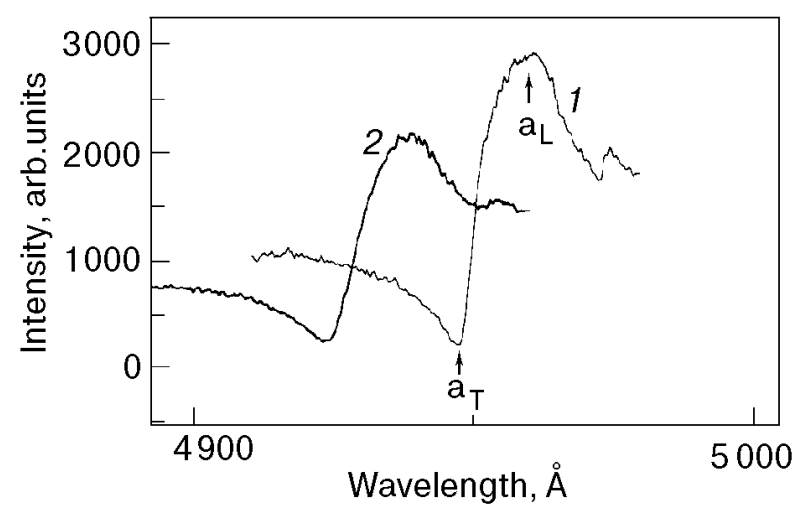

Fig. 1. Reflection spectra of $\mathrm{PbI}_{2}$ single crystals before (1) and after (2) ultrasonic irradiation; $a_{L}, a_{T}-$ upper and lower polaritonic branches, correspondigly; $T=1.4 \mathrm{~K}$.

or of the AE signal is explained by the changes of a dislocation structure inside the sample [3].

An interesting result was obtained when measuring the low temperature $(T=1.4 \mathrm{~K})$ photoluminescence spectra from a natural crystal surface. In these spectra the localized exciton line disappeared after the ultrasonic irradiation. This fact can be explained by the considerable increase of the nearsurface nonradiative recombination rate caused by a migration of defects to the surface of the sample.

In our experiments we have observed for the first time an acoustically stimulated phase transition. It

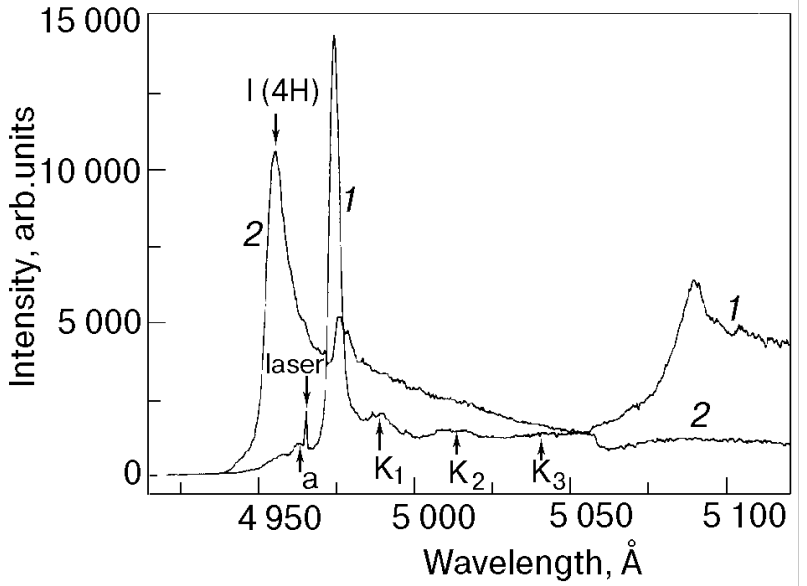

Fig. 2. Photoluminescence spectra of $\mathrm{PbI}_{2}$ single crystals before (1) and after (2) ultrasonic irradiation; $a-$ free excition, $I-$ localized excition, $K_{1}, K_{2}, K_{3}-K$-series. $T=1.4 \mathrm{~K}$.

is shown that a dislocation structure is changing under the ultrasonic irradiation. The high stability of the obtained $4 H$ polytype is of special interest. These fact and acoustic emission anomalies are under discussion.

1. R. S. Mitchell, Z. Krist. 111, 372 (1959).

2. N. M. Belyi, A. V. Bobyr, E. A. Vinogradov, I. S. Gorban', V. A. Gubanov, and G. N. Zhyzhyn, Fiz. Tverd. Tela 24, 887 (1982).

3. V. S. Boiko, R. I. Garber, V. F. Kivshyk, and L. F. Krivenko, Fiz. Tverd. Tela 17, 1541 (1975). 